\title{
The harmonic probing method for output-only nonlinear mechanical systems
}

\author{
Oscar Scussel $^{1} \cdot$ Samuel da Silva $^{1}$
}

Received: 2 August 2016 / Accepted: 20 January 2017 / Published online: 6 February 2017

(C) The Brazilian Society of Mechanical Sciences and Engineering 2017

\begin{abstract}
Most engineering applications involving vibrating structures are nonlinear in nature and many techniques have been recently investigated to provide a better understanding of such problems. Among the large variety of methods, the harmonic probing has presented useful properties for identification and dynamic analysis of nonlinear systems. The method is conventionally described by the multi-dimensional Fourier transform of the Volterra kernels and it depends on the knowledge of the equations of motion and the respective input and output data. However, this white-box methodology is limited to applications where the input signal is either unknown or even unmeasured. Thus, the present paper is concerned with the development of an extended version of the harmonic probing method to deal with applications where only the outputs are available. The algebraic expressions of the extended Volterra kernels transform and their theoretical properties are provided. The main advantages, novelties and drawbacks of this new approach are discussed and compared with the conventional approach. It is verified that the new kernels can be expressed as a combination of the conventional ones. Numerical tests based on a classical 2DOF Duffing oscillator are carried out and the results reveal the effectiveness and potential of the extended harmonic probing method based on a nonparametric model using new kernels
\end{abstract}

Technical Editor: Kátia Lucchesi Cavalca Dedini.

Oscar Scussel

oscar.scussel@gmail.com

Samuel da Silva

samuel@dem.feis.unesp.br

1 Departamento de Engenharia Mecânica, Faculdade de Engenharia de Ilha Solteira, Unesp-Universidade Estadual Paulista, Av. Brasil 56, Ilha Solteira, SP 15385-000, Brazil to describe a prediction of vibrating systems in nonlinear regime of motion.

Keywords Nonlinear dynamics · Harmonic probing · Volterra series · Output-only identification

\section{Introduction}

The development of methods to analyse and understand nonlinear vibrations has been a topic of interest during recent years in signal processing and in structural dynamics applications. Normally, vibrating engineering structures can present a wide variety of complex and interesting effects involving geometrical nonlinearities, joints and gaps or even properties of the material nonlinearities and many others $[16,33]$. The identification of problems characterized by such complex effects becomes a difficult challenge once the algebraic solution is not well established and does not exist in some cases. Among the several existing methods to deal with this kind of problem, the Volterra series representation has been extensively used with satisfactory results $[22,25,26,35]$. The Volterra series allows the output of nonlinear systems to be represented as a polynomial power series expansion in the time domain through the multi-dimensional convolutions between the input signal and the higher-order Volterra kernels [23]. Recently, Cheng et al. [11] presented a state-of-the-art review of the Volterra series and their powerful benefits and broad applications in many different fields of engineering science such as biomedical engineering, fluid dynamics, electrical engineering, mechanical engineering, etc.

The multi-dimensional Fourier transform of the Volterra kernels generates higher-order frequency response functions (HOFRFs) that are used to describe the conventional 
harmonic probing method $[2-4,6]$. The harmonic probing algorithm is simply an extension of the usual procedure for obtaining the linear transfer functions. It is a white-box modelling approach and the solution is computed using the Fourier transform of the Votlerra kernels. These functions are very useful and have been extensively applied in several problems of structural dynamics. For instance, Cafferty and Tomlinson [5] applied the frequency domain technique using HOFRFs to investigate the energy transfer properties and to characterize an application involving a Monroe automotive damper. Furthermore, HOFRFs have been used in applications of modal testing [27, 29].

Storer and Tomlinson [27] showed that the higherorder transfer functions up to third-order can be measured through sine excitation on a practical case involving a nonlinear beam rig. The paper illustrated the possibility of detecting nonlinear effects by using the HOFRFs. However, the method studied suffers some limitations once the HOFRFs cannot be well measured due to the bending resonant peaks and it requires curve-fitting procedures. Additionally, Tawfiq and Vinh [29] found that HOFRFs as well as the Volterra kernels constitute the bases of nonlinear modal analysis. The utilization of the quotient of polynomials for transfer functions and impulse response functions revealed a great importance allowing interpreting physical phenomena (damping, eigenvalues, etc.). Many researchers have investigated the harmonic probing algorithm for nonlinear system analysis for single-inputs to understand the phenomena of harmonics and nonlinear behaviour [3, 4]. However, in Worden et al. [34] an extension of the harmonic probing method is proposed for both continuous and discrete-time subject to multiple sinusoidal inputs. The algebraic expressions revealed that the higher-order and the cross-kernel transforms are directly related with the firstorder kernel and are functions of the nonlinear parameters via polynomial representation.

Although this representation provides useful tool for the study of nonlinear behaviour, it may have convergence problems [8, 21, 30, 31]. Tomlinson et al. [31] proposed a criterion to determine the upper limit to the sinusoidal excitation level at the natural frequency. This strategy provides an estimation of the magnitude amplitude at which the output starts to diverge. The results obtained through single degree-of-freedom (SDOF) and 2DOF Duffing oscillators with cubic stiffness revealed the accuracy of the method to establish the magnitude of the harmonic input and the number of terms required in the Volterra series expansion to predict the response of systems described by polynomial nonlinearities. In addition, Thouverez [30] provided an alternative procedure to estimate an upper limit of the convergence radius of the Volterra series by using the dynamical equations in terms of the harmonic balance method. Furthermore, the complex variable properties with Rouch's theorem and Lagrange's expansion are applied to obtain the convergence criterion. The examples using Duffing oscillators with nonlinear springs showed that the approximation due to the harmonic balance approach does not affect the estimation of the convergence radius. An improved method to analyse the convergence threshold of the Volterra series response was proposed by Chatterjee and Vyas [8]. The contribution lies in a procedure based on critical values of the nondimensional nonlinear parameters for the response convergence over a wide range of excitation frequency. This critical limit value is described as function of the number of the Volterra series terms that added to the approximation of the response predicted. The results based on a 2DOF nonlinear system showed that the critical values are found to be directly dependent on the nonlinear stiffness as well as on the linear coupling stiffness coefficients.

Additionally, the HOFRFs via the harmonic probing method have been extensively used in nonlinear parameter estimation problems. Lee [18] proposed a method to compute the higher-order kernel transforms using component separation technique that allows the response component of first-order to be extracted. The nonlinear parameters were estimated by a relationship between the first-order and the higher-order kernel transforms. The nonlinear parameter estimation method was applied in simulated systems with nonlinear stiffness and nonlinear damping. The method presented a few disadvantages and convergence problems of the response predicted under high level of amplitude excitation.

Chatterjee and Vyas [9] proposed an alternative approach where the nonlinear parameters along with the first-order kernel transforms are obtained by using a recursive iteration technique. The number of terms of the response series and the excitation amplitude over a wide range of frequencies are driven by the convergence criterion. The procedure is investigated through numerical simulation involving a Duffing oscillator and the robustness is also tested against random measurement noise. This recursive iteration procedure proposed by Chatterjee and Vyas [9] was extended for multi-degrees-of-freedom systems with multiple inputs based on higher-order and cross-kernel transforms by Chatterjee and Vyas [10]. The method was tested through numerical simulation for a rotor-bearing system characterized by quadratic and cubic stiffness nonlinearities. Convergence and error analysis were also carried out and the results illustrated the accuracy of the method for nonlinear system analysis under multi-input harmonic excitations. Recently, Chatterjee [7] applied the HOFRF for damage detection problems on nonlinear response characteristics of a cracked beam. This study investigated the use of the Volterra series for developing a quantitative damage assessment for a benchmark composed by a cantilever beam with edge crack. 
Although the conventional harmonic probing method is a useful tool for structural dynamics problems, this tool requires the knowledge of the equations of motion, known as white-box modeling. Moreover, the input and output data must be available. Thus, the analysis in operational conditions becomes very limited in several real-world engineering applications once they are driven by unknown inputs.

In order to overcome these limitations involving problems where the input signal is not directly available or totally unknown in their environmental operating, many concepts and techniques of blind system identification (BSI) have been developed and employed $[1,15]$. A vast number of methods based on blind source separation (BSS) techniques have been widely used due to their potential in modal analysis based on output-only signals [14, 19, 36]. In such cases, the Volterra series theory has been extensively used mainly in areas such as signal processing and communications [12, $24,28]$ and damage detection and location in multi-degreeof-freedom (MDOF) systems based on the transmissibility of nonlinear output frequency response functions (NOFRFs) [17, 37]. Scussel and Silva [24] proposed recently the use of Volterra series and orthonormal basis expansions to identify the higher-order kernels in the discrete-time domain through a least-squares approach and output-only signals. The results based on numerical and experimental applications demonstrated the applicability and benefits of the Volterra-Kautz series expansion. Although these recent blind methods based on Volterra series allow to identify nonlinear systems, it would be very desirable a simple extension of the conventional harmonic probing method in order to deal directly with problems of structural dynamics through the extended HOFRFs and the respective nonlinear parameters in the continuous time domain.

In this context, the present paper is aimed at proposing a new version of the conventional harmonic probing method based on the following contributions:

(i) A new theorem is derived to prove the existence of the extended HOFRFs for continuous nonlinear systems.

(ii) An alternative procedure based on the conventional harmonic probing method is presented to obtain the analytical expressions of the extended HOFRFs.

(iii) Theoretical aspects of the model based on output-only as well as its significance are addressed.

The main advantage of this new method lies in its ability to treat nonlinearities in applications where only the output signals are available.

The layout of the present work is organized as follows: Section 2 reviews briefly the Volterra series representation and the harmonic probing algorithm for single-input and multi-output (SIMO) systems. The algebraic expressions of the extended HOFRFs based on output-only data

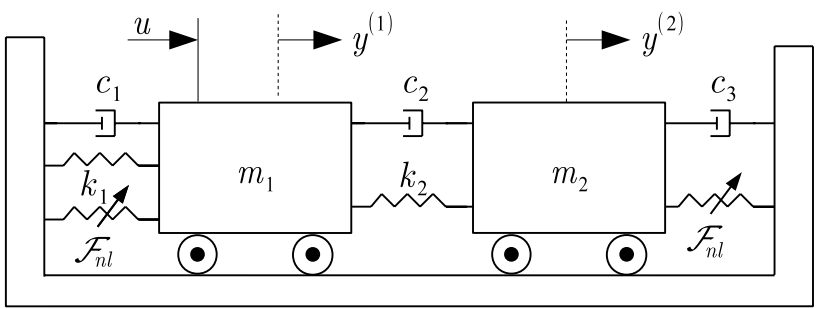

Fig. 1 Duffing oscillator with 2DOF and nonlinearities involving quadratic and cubic stiffnesses

are presented along Sect. 3. In Sect. 4 a numerical example based on a 2DOF Duffing oscillator is carried out and comparisons with the conventional harmonic probing method results are made. Finally, the conclusions are summarized in Sect. 5.

\section{Volterra series and harmonic probing method}

The Volterra series is a powerful way to describe the relationship of input and output of nonlinear systems through multi-dimensional convolutions. For single-input and multi-output (SIMO) systems, the functionals of the Volterra series relate the output measured at the $p$-th point with the following input [23]:

$y^{(p)}(t)=\sum_{\eta=1}^{+\infty} y_{\eta}^{(p)}(t)=y_{1}^{(p)}(t)+y_{2}^{(p)}(t)+y_{3}^{(p)}(t)+\cdots$,

where the respective polynomial contributions of $\eta$ th order are given by the following mapping:

$y_{\eta}^{(p)}(t)=\int_{-\infty}^{+\infty} \cdots \int_{-\infty}^{+\infty} h_{\eta}^{(p)}\left(\tau_{1}, \ldots, \tau_{\eta}\right) \prod_{i=1}^{\eta} u\left(t-\tau_{i}\right) \mathrm{d} \tau_{i}$.

The term $h_{\eta}^{(p)}\left(\tau_{1}, \ldots, \tau_{\eta}\right)$ is called the Volterra kernel of $\eta$ th order related with the $p$ th output and is a generalization of the well-known impulse response function (IRF). The multi-dimensional Fourier transform of the Volterra kernels can be calculated by

$$
\begin{aligned}
\mathcal{H}_{\eta}^{(p)}\left(\omega_{1}, \ldots, \omega_{\eta}\right)= & \int_{-\infty}^{+\infty} \cdots \int_{-\infty}^{+\infty} h_{\eta}^{(p)}\left(\tau_{1}, \ldots, \tau_{\eta}\right) \\
& \times \prod_{i=1}^{\eta} e^{-j \omega_{i} \tau_{i}} \mathrm{~d} \tau_{1} \cdots \mathrm{d} \tau_{\eta} .
\end{aligned}
$$

Now, by considering the property of symmetry of the kernels, as can be found in Cafferty and Tomlinson [5], and a single-tone input $u(t)=\frac{A}{2} e^{j \omega t}+\frac{A}{2} e^{-j \omega t}$, the output in Eq. (1) is given as follows: 


$$
\begin{aligned}
y^{(p)}(t)= & \frac{A}{2} \mathcal{H}_{1}^{(p)}(\omega) e^{j \omega t}+\frac{A}{2} \mathcal{H}_{1}^{(p)}(-\omega) e^{-j \omega t} \\
& +\frac{A^{2}}{4} \mathcal{H}_{2}^{(p)}(\omega, \omega) e^{j 2 \omega t}+\frac{A^{2}}{2} \mathcal{H}_{2}^{(p)}(-\omega, \omega)+\frac{A^{2}}{4} \mathcal{H}_{2}^{(p)}(-\omega,-\omega) e^{-j 2 \omega t} \\
& +\frac{A^{3}}{8} \mathcal{H}_{3}^{(p)}(\omega, \omega, \omega) e^{j 3 \omega t}+\frac{3 A^{3}}{8} \mathcal{H}_{3}^{(p)}(-\omega, \omega, \omega) e^{j \omega t} \\
& +\frac{3 A^{3}}{8} \mathcal{H}_{3}^{(p)}(-\omega,-\omega, \omega) e^{-j \omega t}+\frac{A^{3}}{8} \mathcal{H}_{3}^{(p)}(-\omega,-\omega,-\omega) e^{-j 3 \omega t} \\
& +\cdots
\end{aligned}
$$

where $A$ is the forcing level amplitude and $\omega$ is the frequency of excitation.

Without loss of generality, a classical two degrees-offreedom (2DOF) Duffing oscillator, as can be seen in Fig. 1, with quadratic and cubic nonlinearity is used to illustrate the method. Furthermore, at the end of this section the framework of the conventional HOFRFs as a function of the physical parameters of mass, damping and stiffness is reviewed.

The system in Fig. 1 is described by

$\mathbf{M} \ddot{\mathbf{y}}+\mathbf{C} \dot{\mathbf{y}}+\mathbf{K y}+\mathcal{F}_{n l}(\mathbf{y})=\mathbf{u}$,

where $\mathbf{M}, \mathbf{C}$ and $\mathbf{K}$ are the mass, damping and stiffness matrices, respectively, given by

$$
\begin{gathered}
\mathbf{M}=\left[\begin{array}{cc}
m_{1} & 0 \\
0 & m_{2}
\end{array}\right], \quad \mathbf{K}=\left[\begin{array}{cc}
k_{1}+k_{2} & -k_{2} \\
-k_{2} & k_{2}
\end{array}\right] \\
\text { and } \quad \mathbf{C}=\left[\begin{array}{cc}
c_{1}+c_{2} & -c_{2} \\
-c_{2} & c_{2}+c_{3}
\end{array}\right] .
\end{gathered}
$$

The term $\mathbf{u}$ is the force excitation vector and $\mathbf{y}$ is the vector of outputs:

$\mathbf{u}=\{u(t) 0\}^{T}, \mathbf{y}=\left\{y^{(1)}(t) y^{(2)}(t)\right\}^{T}$,

where the superscript ${ }^{T}$ denotes the transpose and $\mathcal{F}_{\text {nl }}(\mathbf{y})$ denotes the nonlinear functional given by

$\mathcal{F}_{\text {nl }}(\mathbf{y})=\left\{\alpha_{1}\left[y^{(1)}(t)\right]^{2}+\beta_{1}\left[y^{(1)}(t)\right]^{3} \alpha_{2}\left[y^{(2)}(t)\right]^{2}+\beta_{2}\left[y^{(2)}(t)\right]^{3}\right\}^{T}$.

From the Volterra expansion in Eq. (1) with $\eta=3$, the displacement of the first mass can be represented by

$$
\begin{aligned}
y^{(1)}(t)= & \underbrace{\int_{-\infty}^{\infty} h_{1}^{(1)}\left(\tau_{1}\right) u\left(t-\tau_{1}\right) \mathrm{d} \tau_{1}}_{y_{1}^{(1)}(t)} \\
& +\underbrace{\int_{-\infty}^{\infty} \int_{-\infty}^{\infty} h_{2}^{(1)}\left(\tau_{1}, \tau_{2}\right) u\left(t-\tau_{1}\right) u\left(t-\tau_{2}\right) \mathrm{d} \tau_{1} \mathrm{~d} \tau_{2}}_{y_{2}^{(1)}(t)} \\
& +\underbrace{\int_{-\infty}^{\infty} \int_{-\infty}^{\infty} \int_{-\infty}^{\infty} h_{3}^{(1)}\left(\tau_{1}, \tau_{2}, \tau_{3}\right) u\left(t-\tau_{1}\right) u\left(t-\tau_{2}\right) u\left(t-\tau_{3}\right) \mathrm{d} \tau_{1} \mathrm{~d} \tau_{2} \mathrm{~d} \tau_{3}}_{y_{3}^{(1)}(t)},
\end{aligned}
$$

where $y_{1}^{(1)}(t)$ is the linear contribution, $y_{2}^{(1)}(t)$ is the quadratic contribution and $y_{3}^{(1)}(t)$ denotes the cubic polynomial contribution. Assuming a harmonic input $u(t)=A e^{j \omega t}$ applied to the first mass, the output can be rewritten as a function of the HOFRFs:

$$
\begin{aligned}
y^{(1)}(t)= & A e^{j \omega t} \mathcal{H}_{1}^{(1)}(\omega)+A^{2} e^{j 2 \omega t} \mathcal{H}_{2}^{(1)}(\omega, \omega) \\
& +A^{3} e^{j 3 \omega t} \mathcal{H}_{3}^{(1)}(\omega, \omega, \omega) .
\end{aligned}
$$

Differentiating $y^{(1)}(t)$ for the velocity and the acceleration yields

$$
\begin{aligned}
\dot{y}^{(1)}(t)= & j \omega A e^{j \omega t} \mathcal{H}_{1}^{(1)}(\omega)+j 2 \omega A^{2} e^{j 2 \omega t} \mathcal{H}_{2}^{(1)}(\omega, \omega) \\
& +j 3 \omega A^{3} e^{j 3 \omega t} \mathcal{H}_{3}^{(1)}(\omega, \omega, \omega), \\
\ddot{y}^{(1)}(t)= & -\omega^{2} A e^{j \omega t} \mathcal{H}_{1}^{(1)}(\omega)-4 \omega^{2} A^{2} e^{j 2 \omega t} \mathcal{H}_{2}^{(1)}(\omega, \omega) \\
& -9 \omega^{2} A^{3} e^{j 3 \omega t} \mathcal{H}_{3}^{(1)}(\omega, \omega, \omega) .
\end{aligned}
$$

By substituting the terms $y^{(1)}(t), \dot{y}^{(1)}(t)$ and $\ddot{y}^{(1)}(t)$ into the Eq. (5) and equating the coefficients of $A e^{j \omega t}$ generates the following expressions for the FRFs of the first order:

$\mathcal{H}_{1}^{(1)}(\omega)=\frac{1}{-m_{1} \omega^{2}+\left(k_{1}+k_{2}\right)+j \omega\left(c_{1}+c_{2}\right)}$,

$\mathcal{H}_{1}^{(2)}(\omega)=\frac{1}{-k_{2}-j \omega c_{2}}$,

where the FRFs of the second order are obtained by equating the coefficients of $A^{2} e^{j 2 \omega t}$ : 
$\mathcal{H}_{2}^{(1)}(\omega, \omega)=-\alpha_{1}\left[\mathcal{H}_{1}^{(1)}(\omega)\right]^{2} \mathcal{H}_{1}^{(1)}(2 \omega)$,

Now, equating the coefficients of $A^{3} e^{j 3 \omega t}$ yields the FRF of the third order:

$\mathcal{H}_{3}^{(1)}(\omega, \omega, \omega)=\left[\mathcal{H}_{1}^{(1)}(\omega)\right]^{3} \mathcal{H}_{1}^{(1)}(3 \omega)\left[2 \alpha_{1}^{2} \mathcal{H}_{1}^{(1)}(2 \omega)-\beta_{1}\right]$.

Nevertheless, the conventional harmonic probing method is applicable only in cases where the input signal is known. Thus, this formulation is quite limited for applications under operational conditions where the excitation force is unmeasured. Consequently, an extended version of the conventional method to treat such cases through output-only is very welcome. Thus, the Sect. 3 presents the main new contribution of the present work, which an extension of the harmonic probing method and the algebraic expressions of the extended HOFRFs based on output-only data.

\section{Extended HOFRFs based on output-only}

This section presents the analytical expressions and a theorem for the existence of the extended HOFRFs until the third order. A similar result for the discrete-time case based on Volterra-Kautz series was performed recently in Scussel and Silva [24]. However, the Proposition in the presented paper is a new contribution compared to presented in Scussel and Silva [24] because it takes into account the nonparametric framework of the HOFRF in the continuous time domain instead of the parametric structure of the Volterra-Kautz kernels in discrete time domains. An alternative procedure based on the conventional harmonic probing method is presented to obtain the analytical expressions of the extended HOFRFs. Additionally, theoretical aspects of the model based on output-only as well as its significance, benefits and possible limitations are addressed.

Initially, the following assumptions are made:

(i) A harmonic excitation $u(t)=A e^{j \omega t}$ is applied to the first mass in the SIMO system described in Eq. (5).

(ii) Without loss of generality, suppose that the system has a convergent polynomial expansion by using the conventional Volterra kernels truncated up to the third order as in Eq. (7).

Proposition 1 By assuming (i) and (ii), the output signal can be expressed as a multi-dimensional convolution between the extended Volterra kernels and the linear contribution of another output as follows:

$$
\begin{aligned}
y^{(1)}(t)= & \int_{-\infty}^{\infty} g_{1}^{(1)}\left(\tau_{1}\right) y_{1}^{(2)}\left(t-\tau_{1}\right) \mathrm{d} \tau_{1} \\
& +\int_{-\infty}^{\infty} \int_{-\infty}^{\infty} g_{2}^{(1)}\left(\tau_{1}, \tau_{2}\right) y_{1}^{(2)}\left(t-\tau_{1}\right) y_{1}^{(2)}\left(t-\tau_{2}\right) \mathrm{d} \tau_{1} \mathrm{~d} \tau_{2} \\
& +\int_{-\infty}^{\infty} \int_{-\infty}^{\infty} \int_{-\infty}^{\infty} g_{3}^{(1)}\left(\tau_{1}, \tau_{2}, \tau_{3}\right) \\
& \times y_{1}^{(2)}\left(t-\tau_{1}\right) y_{1}^{(2)}\left(t-\tau_{2}\right) y_{1}^{(2)}\left(t-\tau_{3}\right) \mathrm{d} \tau_{1} \mathrm{~d} \tau_{2} \mathrm{~d} \tau_{3}
\end{aligned}
$$

where $g_{1}^{(1)}\left(\tau_{1}\right), \quad g_{2}^{(1)}\left(\tau_{1}, \tau_{2}\right) \quad$ and $g_{3}^{(1)}\left(\tau_{1}, \tau_{2}, \tau_{3}\right)$ are the extended kernels of first, second and third-order, respectively.

Proof Initially, from (i) a harmonic input $u(t)=A e^{j \omega t}$ applied to the first mass of the system in Eq. (5). Thus, from Eq. (7), a linear contribution of the output $y^{(1)}(t)$ is given by

$$
\begin{aligned}
y_{1}^{(1)}(t) & =\int_{-\infty}^{\infty} h_{1}^{(1)}\left(\tau_{1}\right) u\left(t-\tau_{1}\right) \mathrm{d} \tau_{1}=A e^{j \omega t} \mathcal{H}_{1}^{(1)}(\omega) \\
& \Leftrightarrow y_{1}^{(1)}(t)=A e^{j \omega t} \mathcal{H}_{1}^{(2)}(\omega) \mathcal{H}_{1}^{(1)}(\omega)\left[\mathcal{H}_{1}^{(2)}(\omega)\right]^{-1},
\end{aligned}
$$

Assuming that $\mathcal{G}_{1}^{(1)}(\omega)=\mathcal{H}_{1}^{(1)}(\omega)\left[\mathcal{H}_{1}^{(2)}(\omega)\right]^{-1}$ generates

$$
\begin{aligned}
y_{1}^{(1)}(t) & =A e^{j \omega t} \mathcal{H}_{1}^{(2)}(\omega) \mathcal{G}_{1}^{(1)}(\omega) \\
& \Leftrightarrow y_{1}^{(1)}(t)=A e^{j \omega t} \mathcal{H}_{1}^{(2)}(\omega) \int_{-\infty}^{\infty} g_{1}^{(1)}\left(\tau_{1}\right) e^{-j \omega \tau_{1}} \mathrm{~d} \tau_{1} \\
& \Leftrightarrow y_{1}^{(1)}(t)=\int_{-\infty}^{\infty} g_{1}^{(1)}\left(\tau_{1}\right) A e^{j \omega\left(t-\tau_{1}\right)} \mathcal{H}_{1}^{(2)}(\omega) \mathrm{d} \tau_{1} \\
& \Leftrightarrow y_{1}^{(1)}(t)=\int_{-\infty}^{\infty} g_{1}^{(1)}\left(\tau_{1}\right) y_{1}^{(2)}\left(t-\tau_{1}\right) \mathrm{d} \tau_{1} .
\end{aligned}
$$

The quadratic contribution of the output $y^{(1)}(t)$ in Eq. (7) is calculated as:

$$
\begin{aligned}
y_{2}^{(1)}(t)= & A^{2} e^{j 2 \omega t} \mathcal{H}_{2}^{(1)}(\omega, \omega) \\
\Leftrightarrow & y_{2}^{(1)}(t)=A^{2} e^{j 2 \omega t}\left[\mathcal{H}_{1}^{(2)}(\omega)\right]^{2} \\
& {\left[-\alpha_{1}\left[\mathcal{H}_{1}^{(1)}(\omega)\right]^{2} \mathcal{H}_{1}^{(1)}(2 \omega)\right]\left[\mathcal{H}_{1}^{(2)}(\omega)\right]^{-2} } \\
\Leftrightarrow & y_{2}^{(1)}(t)=A^{2} e^{j 2 \omega t}\left[\mathcal{H}_{1}^{(2)}(\omega)\right]^{2} \mathcal{G}_{2}^{(1)}(\omega, \omega) \\
\Leftrightarrow & y_{2}^{(1)}(t)=A^{2} e^{j 2 \omega t}\left[\mathcal{H}_{1}^{(2)}(\omega)\right]^{2} \\
& \times \int_{-\infty}^{\infty} \int_{-\infty}^{\infty} g_{2}^{(1)}\left(\tau_{1}, \tau_{2}\right) e^{-j \omega \tau_{1}} e^{-j \omega \tau_{2}} \mathrm{~d} \tau_{1} \mathrm{~d} \tau_{2} \\
\Leftrightarrow & y_{2}^{(1)}(t)=\int_{-\infty}^{\infty} \int_{-\infty}^{\infty} g_{2}^{(1)}\left(\tau_{1}, \tau_{2}\right) y_{1}^{(2)}\left(t-\tau_{1}\right) y_{1}^{(2)} \\
& \times\left(t-\tau_{2}\right) \mathrm{d} \tau_{1} \mathrm{~d} \tau_{2} .
\end{aligned}
$$

Now, the cubic contribution of $y^{(1)}(t)$ is calculated as follows: 


$$
\begin{aligned}
y_{3}^{(1)}(t)= & A^{3} e^{j 3 \omega t} \mathcal{H}_{3}^{(1)}(\omega, \omega, \omega) \\
\Leftrightarrow & y_{3}^{(1)}(t)=A^{3} e^{j 3 \omega t}\left[\mathcal{H}_{1}^{(2)}(\omega)\right]^{3}\left[\mathcal{H}_{1}^{(1)}(\omega)\right]^{3} \\
& {\left[\mathcal{H}_{1}^{(2)}(\omega)\right]^{-3} \mathcal{H}_{1}^{(1)}(3 \omega)\left[2 \alpha_{1}^{2} \mathcal{H}_{1}^{(1)}(2 \omega)-\beta_{1}\right] } \\
\Leftrightarrow & y_{3}^{(1)}(t)=A^{3} e^{j 3 \omega}\left[\mathcal{H}_{1}^{(2)}(\omega)\right]^{3} \mathcal{G}_{3}^{(1)}(\omega, \omega, \omega) \\
\Leftrightarrow & y_{3}^{(1)}(t)=A^{3} e^{j 3 \omega t}\left[\mathcal{H}_{1}^{(2)}(\omega)\right]^{3} \int_{-\infty}^{\infty} \int_{-\infty}^{\infty} \int_{-\infty}^{\infty} g_{3}^{(1)} \\
& \left(\tau_{1}, \tau_{2}, \tau_{3}\right) e^{-j \omega\left(\tau_{1}+\tau_{2}+\tau_{3}\right)} \mathrm{d} \tau_{1} \mathrm{~d} \tau_{2} \mathrm{~d} \tau_{3} \\
\Leftrightarrow & y_{3}^{(1)}(t)=\int_{-\infty}^{\infty} \int_{-\infty}^{\infty} \int_{-\infty}^{\infty} g_{3}^{(1)}\left(\tau_{1}, \tau_{2}, \tau_{3}\right) y_{1}^{(2)}\left(t-\tau_{1}\right) \\
& y_{1}^{(2)}\left(t-\tau_{2}\right) y_{1}^{(2)}\left(t-\tau_{3}\right) \mathrm{d} \tau_{1} \mathrm{~d} \tau_{2} \mathrm{~d} \tau_{3} .
\end{aligned}
$$

From (ii) implies directly in $y^{(1)}(t)=y_{1}^{(1)}(t)+y_{2}^{(1)}(t)+$ $y_{3}^{(1)}(t)$. Thus, the Proposition 1 is proved.

It is important to emphasize that the output can be predicted alternatively in terms of another output instead of the force excitation. Furthermore, the proof of Proposition 1 can be generalized by considering the extended FRFs $G_{\eta}^{(p)}(\omega, \ldots, \omega)$ for an arbitrary order $\eta$.

Now, a simple procedure with the conventional harmonic probing is applied to obtain the analytical expressions of the extended HOFRFs. From Proposition 1, Eq. (13) can be rewritten as follows:

$$
\begin{aligned}
y^{(1)}(t)= & \int_{-\infty}^{\infty} g_{1}^{(1)}\left(\tau_{1}\right) A e^{j \omega\left(t-\tau_{1}\right)} \mathcal{H}_{1}^{(2)}(\omega) \mathrm{d} \tau_{1} \\
& +\int_{-\infty}^{\infty} \int_{-\infty}^{\infty} g_{2}^{(1)}\left(\tau_{1}, \tau_{2}\right) A e^{j \omega\left(t-\tau_{1}\right)} \mathcal{H}_{1}^{(2)}(\omega) \\
& \times A e^{j \omega\left(t-\tau_{2}\right)} \mathcal{H}_{1}^{(2)}(\omega) \mathrm{d} \tau_{1} \mathrm{~d} \tau_{2} \\
& +\int_{-\infty}^{\infty} \int_{-\infty}^{\infty} \int_{-\infty}^{\infty} g_{3}^{(1)}\left(\tau_{1}, \tau_{2}, \tau_{3}\right) \\
& \times A e^{j \omega\left(t-\tau_{1}\right)} \mathcal{H}_{1}^{(2)}(\omega) A e^{j \omega\left(t-\tau_{2}\right)} \mathcal{H}_{1}^{(2)}(\omega) \\
& \times A e^{j \omega\left(t-\tau_{3}\right)} \mathcal{H}_{1}^{(2)}(\omega) \mathrm{d} \tau_{1} \mathrm{~d} \tau_{2} \mathrm{~d} \tau_{3}, \\
\Leftrightarrow & y^{(1)}(t)=A e^{j \omega(t)} \mathcal{H}_{1}^{(2)}(\omega) \int_{-\infty}^{\infty} g_{1}^{(1)}\left(\tau_{1}\right) e^{-j \omega \tau_{1}} \mathrm{~d} \tau_{1} \\
& +A^{2} e^{j 2 \omega t}\left[\mathcal{H}_{1}^{(2)}(\omega)\right]^{2} \int_{-\infty}^{\infty} \int_{-\infty}^{\infty} \times g_{2}^{(1)} \\
& \left(\tau_{1}, \tau_{2}\right) e^{-j \omega\left(\tau_{1}+\tau_{2}\right)} \mathrm{d} \tau_{1} \mathrm{~d} \tau_{2} \\
& +A^{3} e^{j 3 \omega t}\left[\mathcal{H}_{1}^{(2)}(\omega)\right]^{3} \int_{-\infty}^{\infty} \int_{-\infty}^{\infty} \int_{-\infty}^{\infty} \times g_{3}^{(1)} \\
& \left(\tau_{1}, \tau_{2}, \tau_{3}\right) e^{-j \omega\left(\tau_{1}+\tau_{2}+\tau_{3}\right)} \mathrm{d} \tau_{1} \mathrm{~d} \tau_{2} \mathrm{~d} \tau_{3} .
\end{aligned}
$$

Thus, from Eq. (3) the output is predicted as

$$
\begin{aligned}
y^{(1)}(t)= & A e^{j \omega t} \mathcal{H}_{1}^{(2)}(\omega) \mathcal{G}_{1}^{(1)}(\omega)+A^{2} e^{j 2 \omega t}\left[\mathcal{H}_{1}^{(2)}(\omega)\right]^{2} \mathcal{G}_{2}^{(1)}(\omega, \omega) \\
& +A^{3} e^{j 3 \omega t}\left[\mathcal{H}_{1}^{(2)}(\omega)\right]^{3} \mathcal{G}_{3}^{(1)}(\omega, \omega, \omega) .
\end{aligned}
$$

Differentiating $y^{(1)}(t)$ in Eq. (14) yields expressions for the velocity and acceleration as

$$
\begin{aligned}
\dot{y}^{(1)}(t)= & j \omega A e^{j \omega t} \mathcal{H}_{1}^{(2)}(\omega) \mathcal{G}_{1}^{(1)}(\omega)+j 2 \omega A^{2} e^{j 2 \omega t}\left[\mathcal{H}_{1}^{(2)}(\omega)\right]^{2} \mathcal{G}_{2}^{(1)}(\omega, \omega) \\
& +j 3 \omega A^{3} e^{j 3 \omega t}\left[\mathcal{H}_{1}^{(2)}(\omega)\right]^{3} \mathcal{G}_{3}^{(1)}(\omega, \omega, \omega), \\
\ddot{y}^{(1)}(t)= & -\omega A e^{j \omega t} \mathcal{H}_{1}^{(2)}(\omega) \mathcal{G}_{1}^{(1)}(\omega)-4 \omega^{2} A^{2} e^{j 2 \omega t}\left[\mathcal{H}_{1}^{(2)}(\omega)\right]^{2} \mathcal{G}_{2}^{(1)}(\omega, \omega) \\
& -9 \omega^{2} A^{3} e^{j 3 \omega t}\left[\mathcal{H}_{1}^{(2)}(\omega)\right]^{3} \mathcal{G}_{3}^{(1)}(\omega, \omega, \omega),
\end{aligned}
$$

Substituting the terms $y^{(1)}(t), \dot{y}^{(1)}(t)$ and $\ddot{y}^{(1)}(t)$ into Eq. (5) gives

$$
\begin{aligned}
A e^{j \omega t}= & m_{1}\left[-\omega^{2} A e^{j \omega t} \mathcal{H}_{1}^{(2)}(\omega) \mathcal{G}_{1}^{(1)}(\omega)-4 \omega^{2} A^{2} e^{j 2 \omega t}\right. \\
& {\left[\mathcal{H}_{1}^{(2)}(\omega)\right]^{2} \mathcal{G}_{2}^{(1)}(\omega, \omega) } \\
& \left.-9 \omega^{2} A^{3} e^{j 3 \omega t}\left[\mathcal{H}_{1}^{(2)}(\omega)\right]^{3} \mathcal{G}_{3}^{(1)}(\omega, \omega, \omega)\right] \\
& +\left(k_{1}+k_{2}\right)\left[A e^{j \omega t} \mathcal{H}_{1}^{(2)}(\omega) \mathcal{G}_{1}^{(1)}(\omega)\right. \\
& +A^{2} e^{j 2 \omega t}\left[\mathcal{H}_{1}^{(2)}(\omega)\right]^{2} \mathcal{G}_{2}^{(1)}(\omega, \omega) \\
& \left.+A^{3} e^{j \omega t}\left[\mathcal{H}_{1}^{(2)}(\omega)\right]^{3} \mathcal{G}_{3}^{(1)}(\omega, \omega, \omega)\right] \\
& +\left(c_{1}+c_{2}\right)\left[j \omega A e^{j \omega t} \mathcal{H}_{1}^{(2)}(\omega) \mathcal{G}_{1}^{(1)}(\omega)\right. \\
& +j 2 \omega A^{2} e^{j 2 \omega t}\left[\mathcal{H}_{1}^{(2)}(\omega)\right]^{2} \mathcal{G}_{2}^{(1)}(\omega, \omega) \\
& \left.+j 3 \omega A^{3} e^{j 3 \omega t}\left[\mathcal{H}_{1}^{(2)}(\omega)\right]^{3} \mathcal{G}_{3}^{(1)}(\omega, \omega, \omega)\right] \\
& +\alpha_{1}\left[A e^{j \omega t} \mathcal{H}_{1}^{(2)}(\omega) \mathcal{G}_{1}^{(1)}(\omega)\right. \\
& +A^{2} e^{j 2 \omega t}\left[\mathcal{H}_{1}^{(2)}(\omega)\right]^{2} \mathcal{G}_{2}^{(1)}(\omega, \omega) \\
& \left.+A^{3} e^{j \omega t}\left[\mathcal{H}_{1}^{(2)}(\omega)\right]^{3} \mathcal{G}_{3}^{(1)}(\omega, \omega, \omega)\right]^{2} \\
& +\beta_{1}\left[A e^{j \omega t} \mathcal{H}_{1}^{(2)}(\omega) \mathcal{G}_{1}^{(1)}(\omega)\right. \\
& +A^{2} e^{j 2 \omega t}\left[\mathcal{H}_{1}^{(2)}(\omega)\right]^{2} \mathcal{G}_{2}^{(1)}(\omega, \omega) \\
& \left.+A^{3} e^{j 3 \omega t}\left[\mathcal{H}_{1}^{(2)}(\omega)\right]^{3} \mathcal{G}_{3}^{(1)}(\omega, \omega, \omega)\right]^{3} \\
&
\end{aligned}
$$

The coefficients of $A e^{j \omega t}$ yield:

$$
\begin{aligned}
1 & =\mathcal{G}_{1}^{(1)}(\omega) \mathcal{H}_{1}^{(2)}(\omega)\left[-\omega^{2} m_{1}+\left(k_{1}+k_{2}\right)+\left(c_{1}+c_{2}\right) j \omega\right] \\
& \Leftrightarrow \mathcal{G}_{1}^{(1)}(\omega)=\frac{-k_{2}-c_{2} j \omega}{-\omega^{2} m_{1}+\left(k_{1}+k_{2}\right)+\left(c_{1}+c_{2}\right) j \omega},
\end{aligned}
$$

The extended FRF of first-order in Eq. (15) can be rewritten as

$\mathcal{G}_{1}^{(1)}(\omega)=\frac{\mathcal{H}_{1}^{(1)}(\omega)}{\mathcal{H}_{1}^{(2)}(\omega)}$

Now, equating the coefficients of $A^{2} e^{j 2 \omega t}$ provides

$$
\begin{aligned}
0= & {\left[\mathcal{H}_{1}^{(2)}(\omega)\right]^{2} \mathcal{G}_{2}^{(1)}(\omega, \omega)\left[-4 \omega^{2} m_{1}+\left(k_{1}+k_{2}\right)+\left(c_{1}+c_{2}\right) j 2 \omega\right] } \\
& +\alpha_{1}\left[\mathcal{H}_{1}^{(2)}(\omega) \mathcal{G}_{1}^{(1)}(\omega)\right]^{2} \\
\Leftrightarrow & \mathcal{G}_{2}^{(1)}(\omega, \omega)=-\alpha_{1}\left[\mathcal{G}_{1}^{(1)}(\omega)\right]^{2} \mathcal{H}_{1}^{(1)}(2 \omega),
\end{aligned}
$$


From Eq. (16), the extended FRF of second-order can be expressed as

$\mathcal{G}_{2}^{(1)}(\omega, \omega)=-\alpha_{1}\left[\frac{\mathcal{H}_{1}^{(1)}(\omega)}{\mathcal{H}_{1}^{(2)}(\omega)}\right]^{2} \mathcal{H}_{1}^{(1)}(2 \omega)$.

Furthermore, by equating the coefficients of $A^{3} e^{j 3 \omega t}$ yields

$$
\begin{aligned}
& 0= {\left[\mathcal{H}_{1}^{(2)}(\omega)\right]^{3} \mathcal{G}_{3}^{(1)}(\omega, \omega, \omega) \times\left[-9 \omega^{2} m_{1}+\left(k_{1}+k_{2}\right)+\left(c_{1}+c_{2}\right) j 3 \omega\right] } \\
&+2 \alpha_{1}\left[\mathcal{H}_{1}^{(2)}(\omega)\right]^{3} \mathcal{G}_{1}^{(1)}(\omega) \mathcal{G}_{2}^{(1)}(\omega, \omega)+\beta_{1}\left[\mathcal{H}_{1}^{(2)}(\omega) \mathcal{G}_{1}^{(1)}(\omega)\right]^{3} \\
& \mathcal{G}_{3}^{(1)}(\omega, \omega, \omega)=2 \alpha_{1}^{2} \mathcal{G}_{1}^{(1)}(\omega)^{3} \mathcal{H}_{1}^{(1)}(2 \omega) \mathcal{H}_{1}^{(1)}(3 \omega)-\beta_{1} \mathcal{G}_{1}^{(1)}(\omega)^{3} \mathcal{H}_{1}^{(1)}(3 \omega),
\end{aligned}
$$

Thus, the extended FRF of third-order can be computed as

$$
\mathcal{G}_{3}^{(1)}(\omega, \omega, \omega)=\left[\frac{\mathcal{H}_{1}^{(1)}(\omega)}{\mathcal{H}_{1}^{(2)}(\omega)}\right]^{3} \mathcal{H}_{1}^{(1)}(3 \omega)\left[2 \alpha_{1}^{2} \mathcal{H}_{1}^{(1)}(2 \omega)-\beta_{1}\right] .
$$

Equation (16) shows an important result which describes the extended FRF $\mathcal{G}_{1}^{(1)}(\omega)$ as a simple ratio of the conventional FRFs in Eqs. (9) and (10). Basically, it describes the ratio of two output spectra of the structure. Thus, this new concept of extended FRF presents similar characteristics to the concept of transmissibility once the function $\mathcal{G}_{1}^{(1)}(\omega)$ behaves and it is computed in a similar way as the transmissibility functions (TFs) [13, 20, 32]. The transmissibility is traditionally defined as the ratio of the spectra of two different system outputs and has been widely studied and used for damage detection and fault diagnosis [37].

Moreover, it can be seen in Eqs. (18) and (19) that the second- and third-order extended FRFs, $\mathcal{G}_{2}^{(1)}(\omega, \omega)$ and $G_{3}^{(1)}(\omega, \omega, \omega)$, are also related to the conventional FRFs and the nonlinear parameters as well.

It is important to point out that the extended HOFRFs introduced here have a similar definition as the transmissibility functions of NOFRFs presented by Lang et al. [17] and Zhao [37]. Thus, the extended version of the harmonic probing method may present potential features for further applications in transmissibility for damage detection, location and fault diagnosis of nonlinear systems.

Although the extended HOFRFs reveal great features and benefits for prediction of nonlinear vibrating systems based on output-only, the method might present some drawbacks and limitations such as the convergence of the series, the order of the Volterra kernels and number of parameters to be estimated. Usually, the convergence problems are directly related to the amplitude of excitation, discontinuities and complex nonlinearities. The Volterra series representation might not be appropriate to deal with systems characterized by effects, such as strong jump phenomenon, limit-cycles, chaos, etc $[8,21,30,31]$. In order to deal with problems involving strong nonlinearities it will be necessary to consider more terms in the Volterra series expansion. Despite these limitations discussed, the advantage of the method presented lies in its ability to treat weakly nonlinear systems through the extended HOFRFs up until the third-order. In Sect. 4, the method is applied in a 2DOF system with quadratic and cubic nonlinearities and the results are compared with the conventional harmonic probing method.

\section{Numerical application}

The system in Eq. (5) is used in order to illustrate the approaches by using the conventional and the extended harmonic probing. The main idea is to show that the extended HOFRFs are able to compute the response as well as the conventional HOFRFs. Additionally, the possibility of analysing the polynomial contributions (linear, quadratic and cubic) of the total output predicted is also investigated. The physical parameters $m_{1}=m_{2}=1 \mathrm{~kg}, k_{1}=10^{4}$ $\mathrm{N} / \mathrm{m}, \quad k_{2}=9 \times 10^{4} \mathrm{~N} / \mathrm{m}, \quad c_{1}=c_{2}=c_{3}=10 \quad \mathrm{~N} \quad \mathrm{~s} / \mathrm{m}$, $\alpha_{1}=\alpha_{2}=10^{5} \mathrm{~N} / \mathrm{m}^{2}$ and $\beta_{1}=\beta_{2}=10^{9} \mathrm{~N} / \mathrm{m}^{3}$ are used. The system is characterized by the linear resonances for the first and second mode close to 11 and $68.5 \mathrm{~Hz}$, respectively.

A harmonic input $u(t)=A \cos (2 \pi \omega t)$ with amplitude of $A=0.48 \mathrm{~N}$ and the excitation frequency close to $\omega=11$ $\mathrm{Hz}$ is applied to the first mass. The outputs are obtained by solving numerically the equations of motion through the Newmark method with the Newton-Raphson procedure and initial conditions in $\left(y^{(1)}(0), y^{(2)}(0)\right)=(0,0)$ and $\left.\dot{y}^{(1)}(0), \dot{y}^{(2)}(0)\right)=(0.0240,0.0240)$ in order to obtain the transient and steady-state regimes in the response apparently with the same amplitude. A sampling rate of $500 \mathrm{~Hz}$ that corresponds approximately to 45 times the first fundamental resonance and 8 times the second one is used to avoid aliasing effects.

The simulation is performed with a time duration of 10 $\mathrm{s}$ and using 5001 samples. In addition, the power spectral density (PSD) function of the reference data is calculated. Welch's periodogram is used with a Hanning window over a frequency range of $0-50 \mathrm{~Hz}$. However, in Fig. 2 is shown a zoom of the PSDs over a frequency range of 5-40 Hz to provide a better visual inspection of the curves. Figure 2a shows the PSD of the force excitation, denoted as $P_{u}(\omega)$, where it is possible to note the peak that correspond to the first fundamental frequency of resonance at approximately $11 \mathrm{~Hz}$. Furthermore, in Fig. $2 \mathrm{~b}$ is showed the PSD of the first mass displacement, denoted as $P_{y^{(1)}}(\omega)$ is shown. It can be seen in such curve the presence of even and odd harmonics at 22 and $33 \mathrm{~Hz}$, respectively. These harmonics in the output spectrum point out the nonlinear effects of the system characterized by quadratic and cubic stiffnesses. 

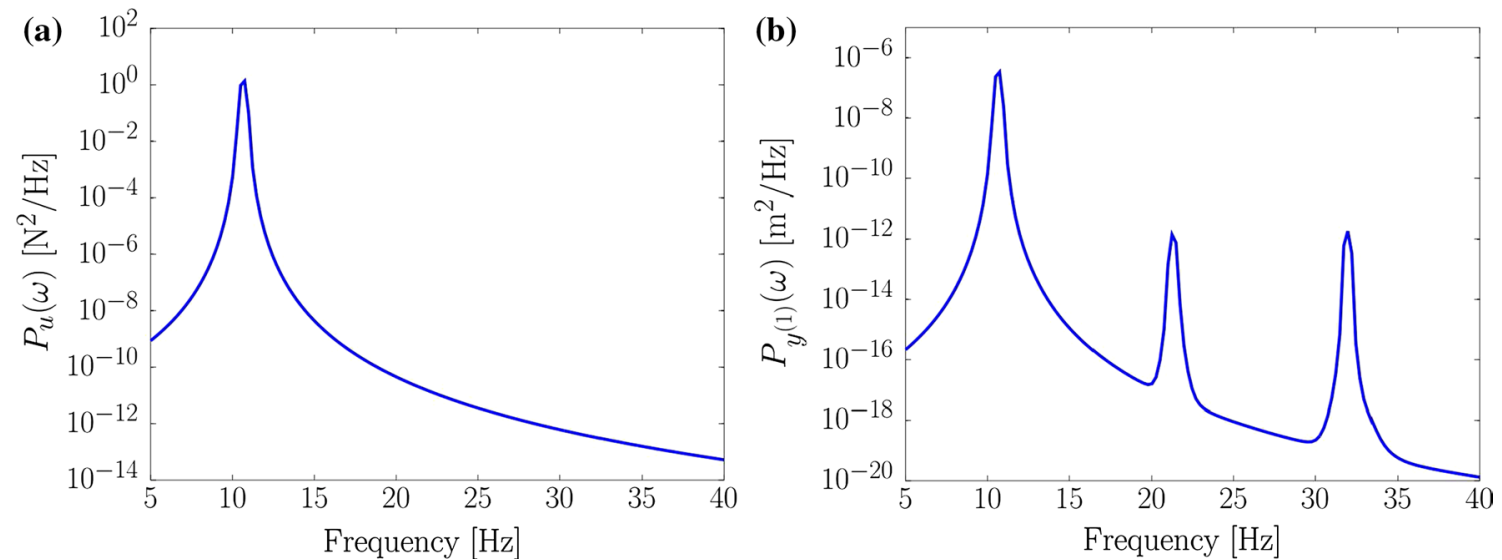

Fig. 2 Analysis of the harmonics in the response signal $y^{(1)}(t)$ when is applied a sinusoidal input on the first mass with excitation close to $11 \mathrm{~Hz}$ and amplitude of $0.48 \mathrm{~N}$. a PSD of the harmonic input applied on the first mass. b PSD of the output $y^{(1)}(t)$ (displacement of the first mass)
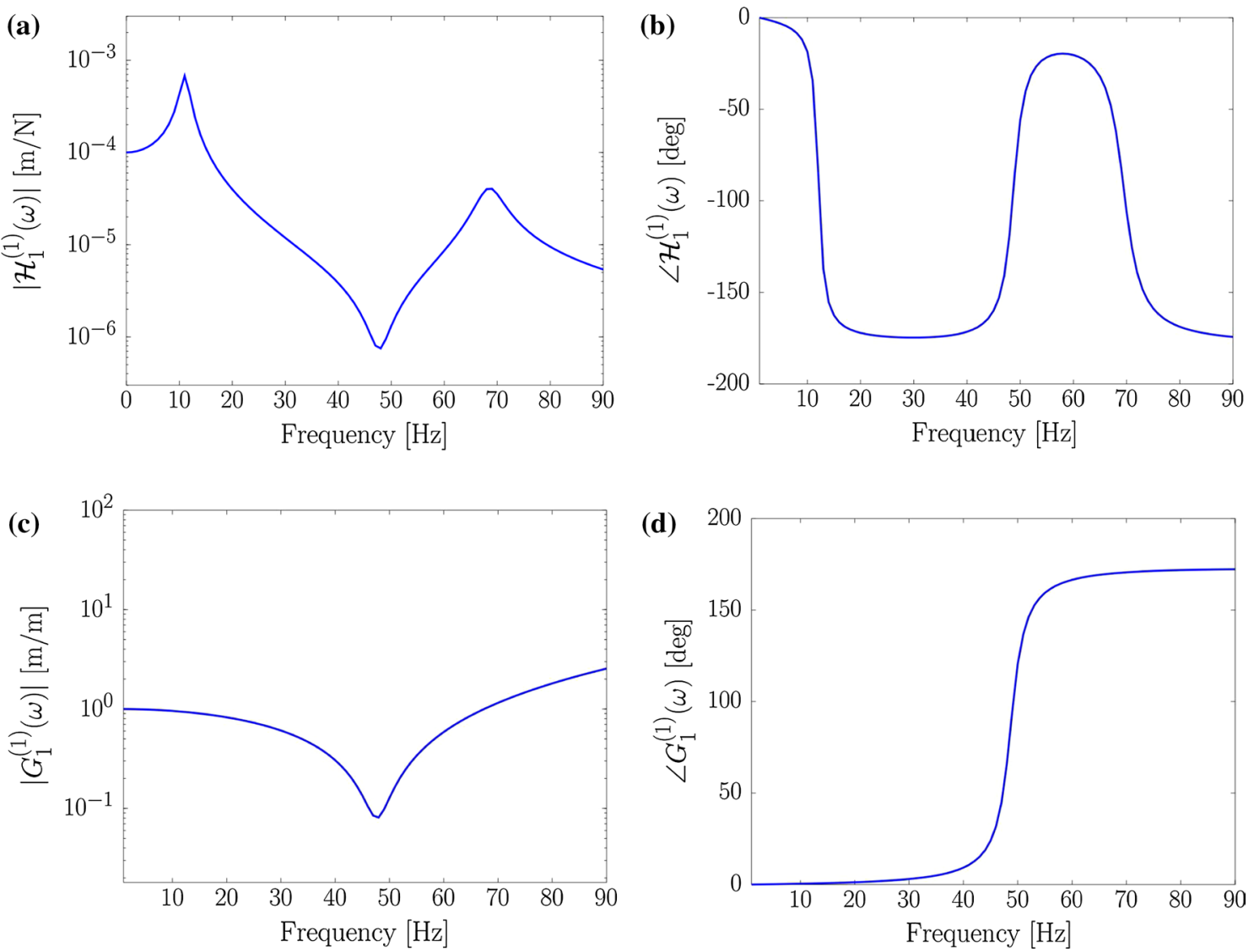

Fig. 3 First-order frequency response functions. a Amplitude of $\mathcal{H}_{1}^{(1)}(\omega)$. b Phase of $\mathcal{H}_{1}^{(1)}(\omega)$. c Amplitude of $\mathcal{G}_{1}^{(1)}(\omega)$. d Phase of $\mathcal{G}_{1}^{(1)}(\omega)$

The physical parameters were used to compute the FRF of first-order $\mathcal{H}_{1}^{(1)}(\omega)$ from Eq. (9) and $\mathcal{G}_{1}^{(1)}(\omega)$ from Eq. (15) over a frequency range of 0-90 with increment step of $0.1 \mathrm{~Hz}$. In general, Fig. 3 shows the comparison between the conventional FRF and extended FRF of first-order.
Figure $3 \mathrm{a}, \mathrm{b}$ shows the magnitude and phase of the FRF $\mathcal{H}_{1}^{(1)}(\omega)$. The resonances peaks of the first mode $(11 \mathrm{~Hz})$ and the second mode $(68.5 \mathrm{~Hz})$ associated with the linear dynamic of the model can be seen. Figure $3 c, d$ shows the magnitude and phase of the extended FRF $\mathcal{G}_{1}^{(1)}(\omega)$ as well. 

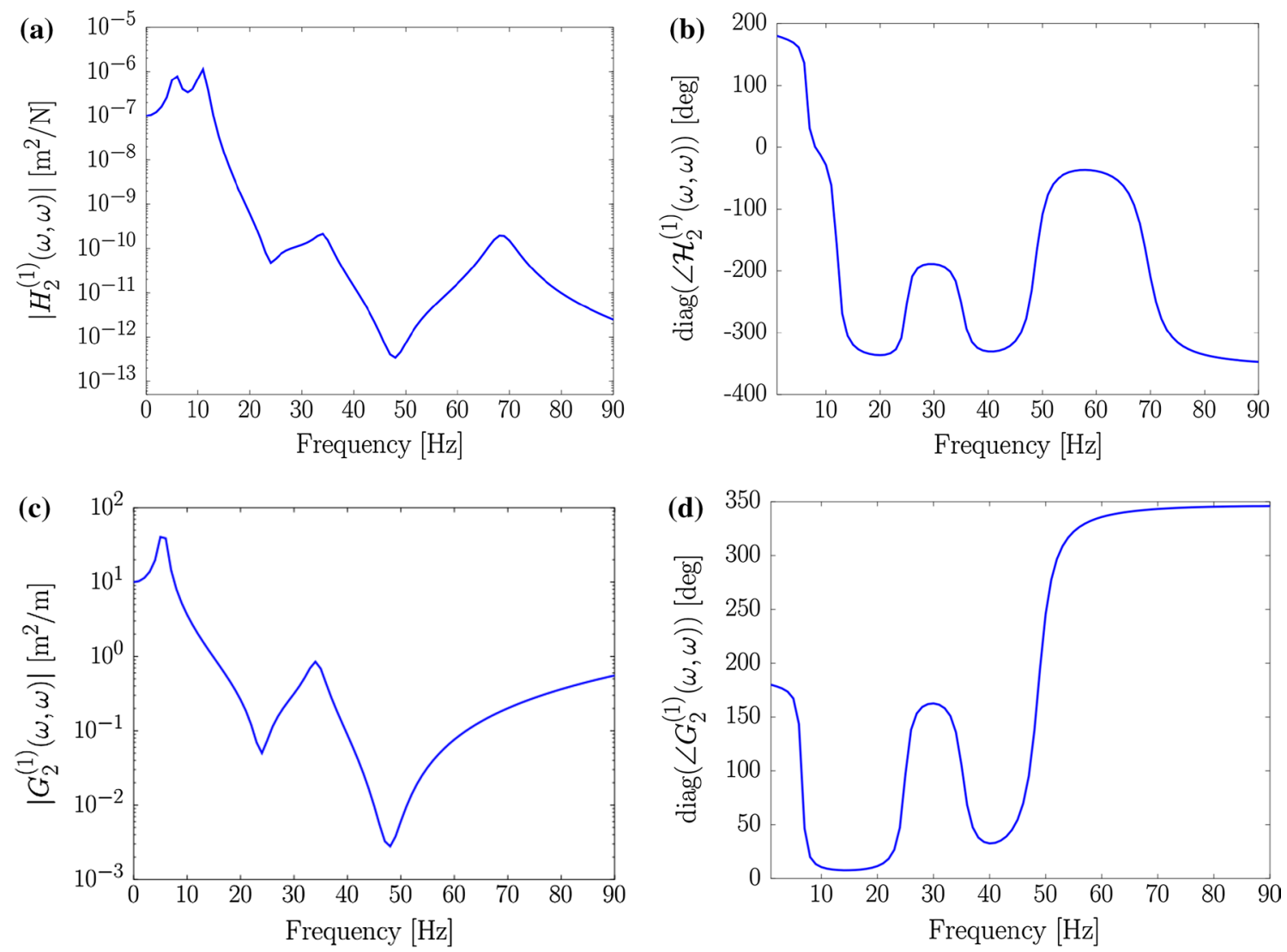

Fig. 4 Principal diagonal of the second-order frequency response functions. a Amplitude of $\mathcal{H}_{2}^{(1)}(\omega, \omega)$. b Phase of $\mathcal{H}_{2}^{(1)}(\omega, \omega)$. c Amplitude of $\mathcal{G}_{2}^{(1)}(\omega, \omega)$. d Phase of $\mathcal{G}_{2}^{(1)}(\omega, \omega)$

In such case, it can be noted that only the anti-resonance peak (close to $48 \mathrm{~Hz}$ ) appears and the figure presents characteristics of the transmissibility curve as can be verified in Eq. (16).

Furthermore, it can be noted that extended FRF and the conventional one differ values in magnitude and phase because of their different framework. Moreover, the extended FRF has an additional term $-k_{2}-c_{2} j$. Although they present different behaviour as discussed, they are used in a similar way to predict the linear contribution $y_{1}^{(1)}(t)$ of the output. While the conventional one is based on the input force excitation $u(t)$, the extended one is based on another output $y_{1}^{(2)}(t)$.

Figure 4 illustrates the principal diagonals of the frequency response functions $\mathcal{H}_{2}^{(1)}(\omega, \omega)$ and $\mathcal{G}_{2}^{(1)}(\omega, \omega)$ in magnitude and phase. It is clearly possible to see in Fig. 4a the resonances at 11 and $68.5 \mathrm{~Hz}$ and their secondary resonances at 5.50 and $34.25 \mathrm{~Hz}$, respectively. Thus, the framework of the conventional FRF of second-order provides information about quadratic nolinearities and is used to compute the quadratic contribution of the total output predicted.
On the other hand, the extended FRF of the second-order contains only the secondary resonances as can be noted in Fig. 4c. This effect happens due to the framework of the function $\mathcal{G}_{2}^{(1)}(\omega, \omega)$ as in Eq. (18).

Figure 5 shows the principal diagonal of the frequency response functions $\mathcal{H}_{3}^{(1)}(\omega, \omega, \omega)$ and $\mathcal{G}_{3}^{(1)}(\omega, \omega, \omega)$ given by Eqs. (12) and (19) in magnitude and phase. The conventional FRF of the third order gives information about cubic nolinearities and is used to compute the cubic contribution of the total output predicted.

In Fig. 5a, it is possible to see that the conventional FRF exhibits the resonances at 11 and $68.5 \mathrm{~Hz}$ and their tertiary resonances at 3.67 and $22.8 \mathrm{~Hz}$, approximately. It is important to note that the secondary resonances do not appear due to the low contribution of the terms of quadratic stiffness. Cafferty and Tomlinson [5] presented an example where the peaks referred to the second order are present in the conventional FRF of the third order once the terms of quadratic stiffness have good contribution.

Moreover, it can be observed in Fig. 5c that the extended FRF which shows that the tertiary resonances 

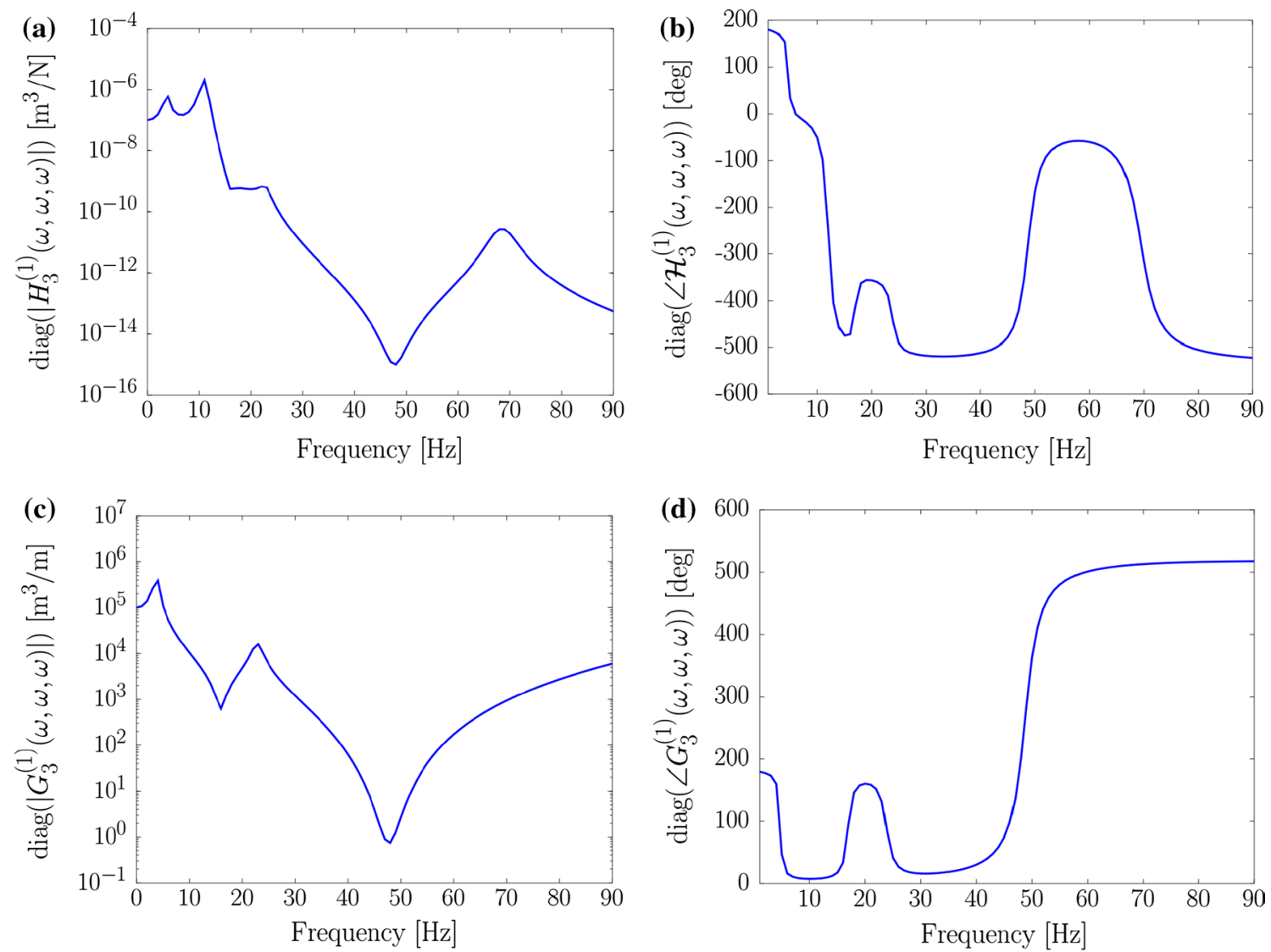

Fig. 5 Principal diagonal of the third-order frequency response functions. a Amplitude of $\operatorname{diag}\left(\mathcal{H}_{3}^{(1)}(\omega, \omega, \omega)\right)$. b Phase of diag $\left(\mathcal{H}_{3}^{(1)}(\omega, \omega, \omega)\right)$. c Amplitude of $\operatorname{diag}\left(\mathcal{G}_{3}^{(1)}(\omega, \omega, \omega)\right)$. d Phase of $\operatorname{diag}\left(\mathcal{G}_{3}^{(1)}(\omega, \omega, \omega)\right)$

are only due the framework of extended HOFRF $\mathcal{G}_{3}^{(1)}(\omega, \omega, \omega)$ from Eq. (19). In general, the extended kernels can be useful in applications of identification and transmissibility of nonlinear systems as discussed at the end of Sect. 3.

The HOFRFs of each method are used to predict the contributions $y_{1}^{(1)}(t), y_{2}^{(1)}(t)$ and $y_{3}^{(1)}(t)$. This fact reveals the main benefits of the Volterra series expansion where it is possible to split the total response predicted in polynomial contributions. Although the framework of the functions $\mathcal{H}_{\eta}^{(p)}\left(\omega_{1}, \ldots, \omega_{\eta}\right)$ and $\mathcal{G}_{\eta}^{(p)}\left(\omega_{1}, \ldots, \omega_{\eta}\right)$ present different behaviour as verified previously, they can be used to predict the response in a similar way. While the conventional one uses the input excitation, the extended one is based on another output measured.

Figure 6 shows the linear, quadratic and cubic contributions of the total output predicted by each method as well as their spectra where it can be noted that there is agreement. In this example, the temporal contribution of the second order is quite low due to the terms $\alpha_{1}$ and $\alpha_{2}$ chosen. Additionally, the asymmetric behaviour of the contribution $y_{2}^{(1)}(t)$ can be noted.
The total output is computed by summing the contributions, $y_{1}^{(1)}(t)+y_{2}^{(1)}(t)+y_{3}^{(1)}(t)$, predicted by the conventional and extended HOFRFs. Figure 7 shows the comparison between the output simulated by the Newmark method (-) and the outputs predicted by the conventional and extended HOFRFs. (*). In both cases, it is possible to see good agreement demonstrating the accuracy of the approaches for output prediction. Additionally, the response spectrum was calculated as shown in Fig. 8. Through the PSD function, it can be noted that the models were able to detect the presence of the fundamental frequency resonance in $11 \mathrm{~Hz}$ and the second and third harmonics, in 22 and 33 $\mathrm{Hz}$, also known as even and odd harmonics.

The results in this work have shown the applicability of the the extended HOFRFs to characterize and detect nonlinear behaviour in vibrating systems. The conventional harmonic probing method is quite illustrative when the differential equations that describe the problem are known analytically. On the other hand, it has been verified that the extended HOFRFs give the same results, but considering the knowledge of the output-only instead input and output as in the conventional case. 

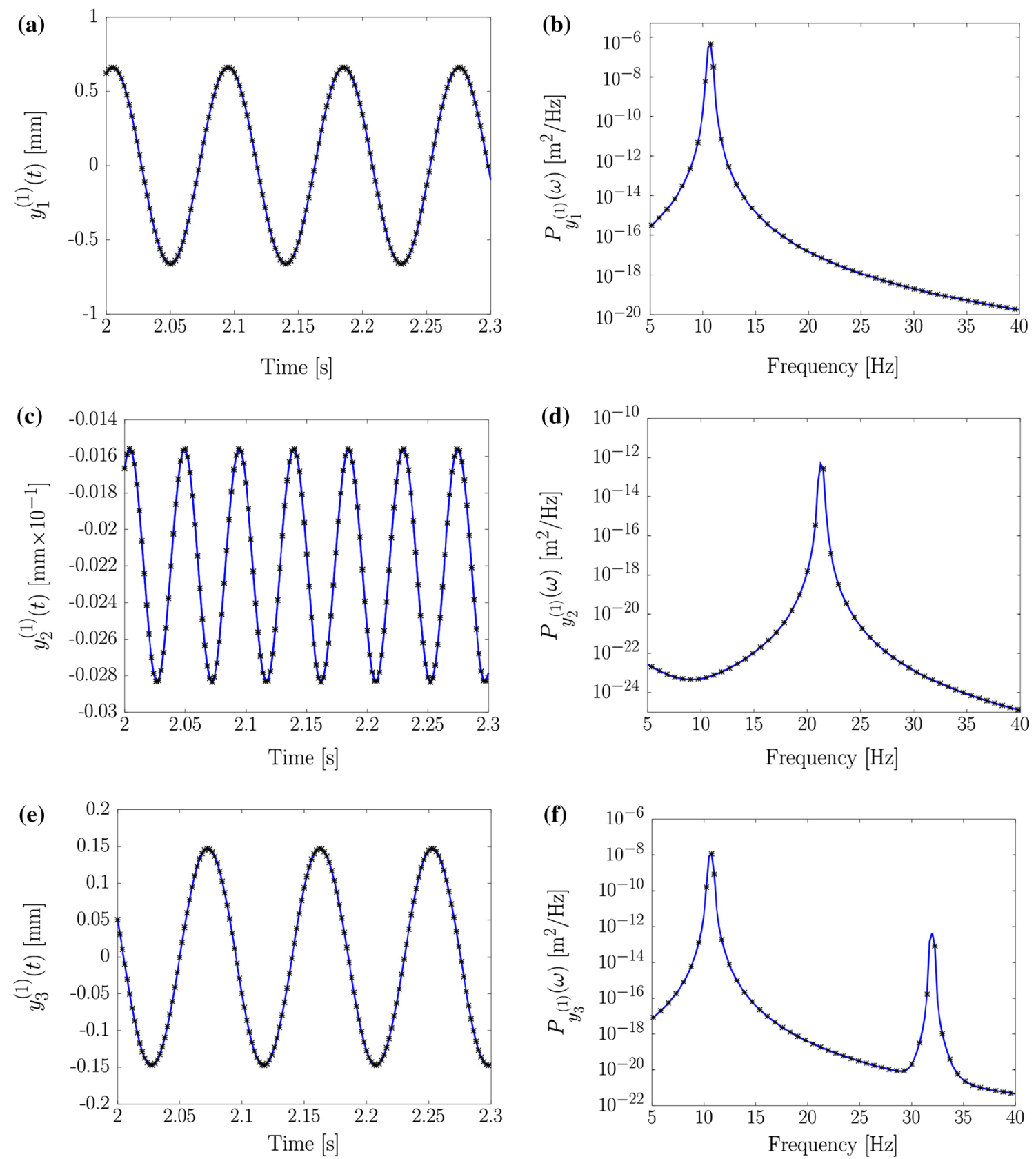

Fig. 6 Comparison between the results obtained using the harmonic probing method (-) and the results reached by the extended harmonic probing method based on output-only data (*). a Linear contribution.

b PSD of the linear contribution. c Quadratic contribution. d PSD of the quadratic contribution. e Cubic contribution. $f$ PSD of the cubic contribution

\section{Final remarks}

In this paper we have presented an extended version of the harmonic probing method. This new approach provides an alternative tool to compute the HOFRFs where only the structural responses are available. The extended HOFRFs can be useful to give information about the nonlinear

behaviour and output prediction. The results obtained using a classical Duffing oscillator with 2DOF characterized by quadratic and cubic stiffnesses have shown the benefits of this new approach for structural dynamics analysis. Furthermore, the method can be applied in nonlinear parameter estimation problems once the new kernels are function of the conventional ones where the 


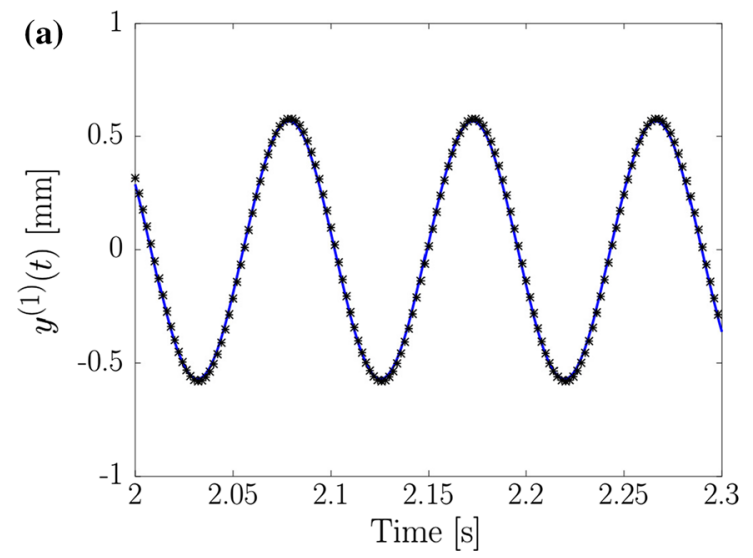

Fig. 7 Comparison of the output simulated by Newmark method () with the outputs predicted by conventional and extended harmonic probing method (*). a Output simulated $(-)$ and predicted by the con-

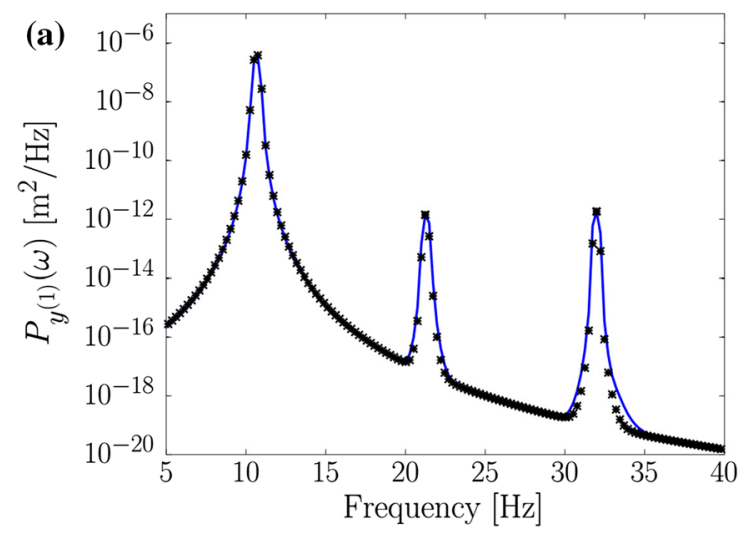

Fig. 8 Comparison of the output spectrum simulated by Newmark method ( - ) with the outputs predicted by the conventional and extended harmonic probing method $(*)$. a Output spectrum simulated

dynamical properties appear clearly. Further applications are concerned with new perspectives on the concept of transmissibility for nonlinear systems based on the extended HOFRFs.

Acknowledgements The authors would like to acknowledge the financial support provided by the São Paulo Research Foundation (FAPESP) by Grant Number 12/09135-3 and National Council for Scientific and Technological Development (CNPq) under Grants Numbers 47058/2012-0 and 203610/2014-8. The first author acknowledges his scholarship from the Coordination for the Improvement of Higher Education Personnel (CAPES). Additionally, the authors would like to thank the anonymous reviewers and professor Michael J. Brennan for their helpful review, relevant comments and useful suggestions.

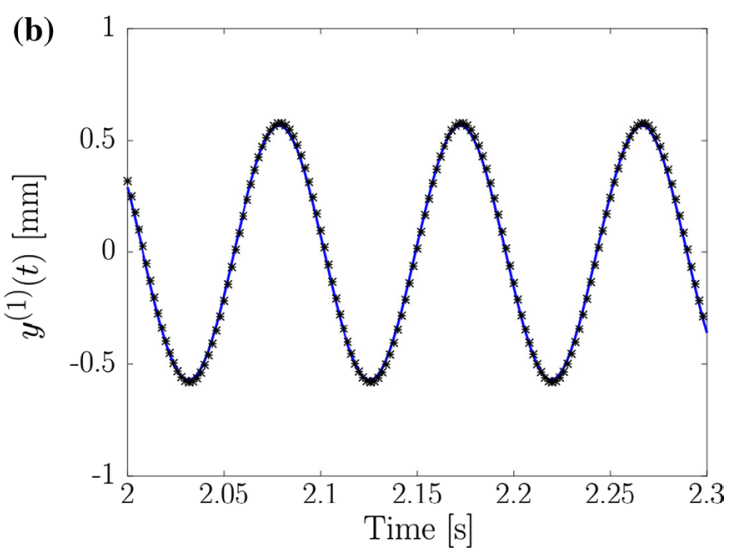

ventional harmonic probing method $(*)$. b Output simulated (-) and predicted by extended harmonic probing method $(*)$

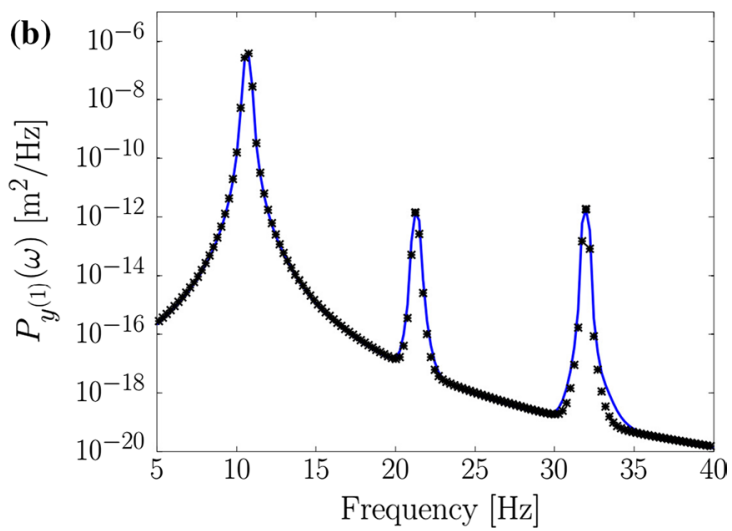

(-) and predicted by conventional harmonic probing method $(*)$. b Output spectrum simulated (-) and predicted by extended harmonic probing method $(*)$

\section{References}

1. Abed-Merain K (1997) Blind system identification. Proc IEEE 85(8):1310-1322. doi:10.1109/5.622507

2. Billings S (2013) Nonlinear system identification: NARMAX methods in the time, frequency, and spatio-temporal domain. Wiley, Chichester, UK

3. Billings S, Tsang K (1989) Spectral analysis for non-linear systems, part I: parametric non-linear spectral analysis. Mech Syst Signal Process 3(4):319-339. doi:10.1016/0888-3270(89)90041-1

4. Billings S, Tsang K (1989) Spectral analysis for non-linear systems, part II: interpretation of non-linear frequency response functions. Mech Syst Signal Process 3(4):341-359. doi:10.1016/0888-3270(89)90042-3

5. Cafferty S, Tomlinson G (1997) Characterization of automotive dampers using higher order frequency response function. 
J Automob Eng 211(3):181-203. http://pid.sagepub.com/content/211/3/181.refs

6. Carassale L, Kareem A (2010) Modeling nonlinear systems by Volterra series. J Eng Mech 136(6):801818. doi:10.1061/(ASCE) EM.1943-7889.0000113

7. Chatterjee A (2010) Structural damage assessment in a cantilever beam with a breathing crack using higher order frequency response functions. J Sound Vib 329(16):3325-3334. doi:10.1016/j.jsv.2010.02.026

8. Chatterjee A, Vyas N (2000) Convergence analysis of Volterra series response of nonlinear systems subjected to harmonic excitation. J Sound Vib 236(2):339-358. doi:10.1006/jsvi.2000.2967

9. Chatterjee A, Vyas NS (2003) Nonlinear parameter estimation in rotor-bearing system using Volterra series and method of harmonic probing. J Vib Acoust 125(3):299-306. doi: $10.1115 / 1.1547486$

10. Chatterjee A, Vyas NS (2004) Non-linear parameter estimation in multi-degree-of-freedom systems using multi-input Volterra series. Mech Syst Signal Process 18(3):457-489. doi:10.1016/ S0888-3270(03)00016-5

11. Cheng C, Peng Z, Zhang W, Meng G (2017) Volterra-seriesbased nonlinear system modeling and its engineering applications: a state-of-the-art review. Mech Syst Signal Process 87:340-364. doi:10.1016/j.ymssp.2016.10.029

12. Cherif I, Abid S, Fnaiech F (2012) Nonlinear blind identification with three-dimensional tensor analysis. Math Probl Eng 2012:22, Article ID 284,815. doi:10.1155/2012/284815

13. Chesné S, Deraemaeker A (2013) Damage localization using transmissibility functions: a critical review. Mech Syst Signal Process 38(2):569-584. doi:10.1016/j.ymssp.2013.01.020

14. Grosel J, Sawicki W, Pakos W (2014) Application of classical and operational modal analysis for examination of engineering structures. Proc Eng 91:136-141. doi:10.1016/j.proeng.2014.12.035. http://www.sciencedirect.com/science/article/ pii/S1877705814030537

15. Kalouptsidis N, Koukoulas P (2003) Blind identification of Volterra-Hammerstein systems. In: Statistical signal processing, 2003 IEEE Workshop, pp 202-205. doi:10.1109/SSP.2003.1289379

16. Kerschen G, Worden K, Vakakis AF, Golinval J (2006) Past, present and future of nonlinear system identification in structural dynamics. Mech Syst Signal Process 20(3):505-592. doi:10.1016/j.ymssp.2005.04.008

17. Lang Z, Park G, Farrar C, Todd M, Mao Z, Zhao L, Worden K (2011) Transmissibility of non-linear output frequency response functions with application in detection and location of damage in MDOF structural systems. Int J Non Linear Mech 46(6):841853. doi:10.1016/j.ijnonlinmec.2011.03.009. http://www.sciencedirect.com/science/article/pii/S0020746211000333

18. Lee G (1997) Estimation of non-linear system parameters using higher-order frequency response functions. Mech Syst Signal Process 11(2):219-228. doi:10.1006/mssp.1996.0080

19. Li D, Ren WX, Hu YD, Yang D (2016) Operational modal analysis of structures by stochastic subspace identification with a delay index. Struct Eng Mech 59(1):187-207. doi:10.12989/ sem.2016.59.1.187

20. Maia NMM, Silva JMM, Ribeiro AMR (2001) The transmissibility concept in multi-degree-of-freedom systems. Mech Syst Signal Process 15(1):129-137. doi:10.1006/mssp.2000.1356
21. Peng Z, Lang Z (2007) On the convergence of the Volterra-series representation of the Duffing's oscillators subjected to harmonic excitations. J Sound Vib 305(12):322-332. doi:10.1016/j. jsv.2007.03.062

22. Rugh WJ (1981) Nonlinear system theory-the Volterra/Wiener approach. University Press, The Johns Hopkings, Baltimore

23. Schetzen M (1980) The Volterra and Wiener theories of nonlinear systems. Wiley, New York

24. Scussel O, da Silva S (2016) Output-only identification of nonlinear systems via Volterra series. ASME J Vib Acoust 138(4):041,012 (13 pages). doi:10.1115/1.4033458

25. Shiki SB, Lopes V Jr, da Silva S (2014) Identification of nonlinear structures using discrete-time Volterra series. J Braz Soc Mech Sci Eng 36(3):523-532. doi:10.1007/s40430-013-0088-9

26. da Silva S (2011) Non-linear model updating of a three-dimensional portal frame based on Wiener series. Int $\mathrm{J}$ Non Linear Mech 46(1):312-320. doi:10.1016/j.ijnonlinmec.2010.09.014

27. Storer D, Tomlinson G (1993) Recent developments in the measurement and interpretation of higher order transfer functions from non-linear structures. Mech Syst Signal Process 7(2):173189. doi: $10.1006 / \mathrm{mssp} .1993 .1006$

28. Tan HZ, Huang Y, Fu J (2008) Blind identification of sparse Volterra systems. Int J Adapt Control Signal Process 22(7):652-662. doi:10.1002/acs.1011

29. Tawfiq I, Vinh T (2003) Contribution to the extension of modal analysis to non-linear structure using Volterra funcitonal series. Mech Syst Signal Process 17(2):379-407. doi:10.1006/ mssp.2002.1499

30. Thouverez F (1998) A new convergence criteria of Volterra series for harmonic inputs. In: IMAC XVI-16th international modal analysis conference, 2-5 February, Santa Barbara, vol 758, pp $723-727$

31. Tomlinson G, Manson G, Lee G (1996) A simple criterion for establishing an upper limit to the harmonic excitation level of the Duffing oscillator using the Volterra series. J Sound Vib 190(5):751-762. doi:10.1006/jsvi.1996.0091. http://www.sciencedirect.com/science/article/pii/S0022460X96900917

32. Weijtjens W, Sitter GD, Devriendt C, Guillaume P (2014) Operational modal parameter estimation of MIMO systems using transmissibility functions. Automatica 50(2):559-564. doi:10.1016/j.automatica.2013.11.021

33. Worden K, Tomlinson GR (2001) Nonlinearity in structural dynamics. Institute of Physics, London

34. Worden K, Manson G, Tomlinson G (1997) A harmonic probing algorithm for the multi-input Volterra series. J Sound Vib 201(1):67-84. doi:10.1006/jsvi.1996.0746

35. Xia X, Zhou J, Xiao J, Xiao H (2016) A novel identification method of Volterra series in rotor-bearing system for fault diagnosis. Mech Syst Signal Process 66-67:557-567. doi:10.1016/j. ymssp.2015.05.006

36. Yang Y, Nagarajaiah S (2013) Output-only modal identification with limited sensors using sparse component analysis. J Sound Vib 332(19):4741-4765. doi:10.1016/j.jsv.2013.04.004

37. Zhao XY, Lang ZQ, Park G, Farrar CR, Todd MD, Mao Z, Worden K (2015) A new transmissibility analysis method for detection and location of damage via nonlinear features in mdof structural systems. IEEE/ASME Trans Mechatron 20(4):19331947. doi:10.1109/TMECH.2014.2359419 